\title{
Tendência de infecções por HIV/Aids: aspectos da ocorrência em idosos entre 2008 e 2018
}

Trend of HIV/AIDS infections: aspects of occurrence in older adults between 2008 and 2018

Tendencia de las infecciones por VIH/SIDA: aspectos de la incidencia en ancianos entre 2008 y 2018

Chrystiany Plácido de Brito Vieira ${ }^{1}$ [D Ana Clara dos Santos e Silva Costa $^{1}$ (])

Maria do Carmo Lopes Dias $^{1}$ (1)

Telma Maria Evangelista de Araújo $^{1}$ (D)

Francisca Tereza de Galiza ${ }^{1}$ (D)

1. Universidade Federal do Piauí. Teresina, PI, Brasil.
Autor correspondente:

Chrystiany Plácido de Brito Vieira.

E-mail: chrystianyplacido@yahoo.com

Recebido em 07/03/2020.

Aprovado em 07/08/2020.

DOI:https://doi.org/10.1590/2177-9465-EAN-2020-0051

\begin{abstract}
Resumo
Objetivo: analisar o perfil sociodemográfico, clínico e epidemiológico dos casos de HIV/Aids em idosos no estado do Piauí. Método: estudo descritivo, com coleta retrospectiva, a partir de dados secundários do Sistema de Informações de Agravos de Notificação, referentes ao período de 2008 a 2018. Coleta realizada de agosto a setembro de 2019, com processamento dos dados realizados por meio do programa TabWin, versão 3.6. Realizou-se cálculo das frequências absoluta e relativa, assim como o cálculo de incidência, utilizando-se do Programa Excel, versão 7.0. Resultados: de 2008 a 2018, notificaram-se 262 casos novos de HIV/Aids em idosos no estado, 4,5\% do número total de casos, com tendência de crescimento no período, sendo $76,0 \%$ da faixa etária entre 60 e 69 anos de idade, $64,9 \%$ do sexo masculino, $64,8 \%$ pardos, $54,5 \%$ com baixa escolaridade, 53,4\% residentes em Teresina e 70,9\% com transmissão pela relação heterossexual. Conclusão e Implicações para a prática: verificou-se tendência de crescimento dos casos de HIV/Aids em idosos, no estado do Piauí, ao longo dos últimos 10 anos. Os resultados deste estudo contribuem para o conhecimento da dinâmica epidemiológica desse agravo no estado e a elaboração de estratégias de prevenção e controle da infecção.
\end{abstract}

Palavras-chave: Idoso; Notificação; HIV; Síndrome de Imunodeficiência Adquirida; Doenças Sexualmente Transmissíveis; Epidemiologia

\begin{abstract}
Objective: this study aimed to analyze the sociodemographic, clinical and epidemiological profile of HIV/AIDS cases in older adults in the state of Piauí, Brazil. Method: this is a descriptive study with retrospective collection, using secondary data from the Brazilian Information System for Notifiable Diseases, from 2008 to 2018. Data collection occurred from August to September 2019 with data processing and mapping performed using the TabWin software, version 3.6. Absolute and relative frequencies were calculated, as well as the incidence, using Microsoft Excel, version 7.0. Results: from 2008 to 2018, there were 262 new cases of HIV/AIDS reported among older adults in the state, representing $4.5 \%$ of the total number of cases, with a growth trend in the period, in which $76.0 \%$ were aged $60-69$ years old, $64.9 \%$ were male, $64.8 \%$ brown-skinned, $54.5 \%$ had low schooling, $53.4 \%$ lived in the city of Teresina, and $70.9 \%$ with transmission through heterosexual relationships. Conclusion and implications for the practice: a growth trend in HIV/AIDS cases in older adults in the state of Piauí over the past 10 years was identified. The results of this study contribute to knowing the epidemiological dynamics of this condition in the state and to developing prevention and control strategies for this infection.
\end{abstract}

Keywords: Aged; Notification; HIV; Acquired Immunodeficiency Syndrome; Sexually Transmitted Diseases; Epidemiology.

\section{Resumen}

Objetivo: analizar el perfil sociodemográfico, clínico y epidemiológico de casos de VIH/SIDA en ancianos del estado de Piauí. Método: estudio descriptivo, con recolección retrospectiva, utilizándose datos secundarios del "Sistema de Informações de Agravos de Notificação", referente al período de 2008 a 2018. Recolección realizada de agosto a septiembre de 2019, con procesamiento de datos realizado en el Programa TabWin, versión 3.6. Se realizó el cálculo de frecuencias absolutas y relativas, así como el cálculo de incidencia, utilizándose del Programa Excel, versión 7.0. Resultados: de 2008 a 2018 se notificaron 262 nuevos casos de VIH/SIDA en ancianos en el estado, $4.5 \%$ del total de casos, con tendencia creciente en el período, siendo $76.0 \%$ entre 60 y 69 años, $64,9 \%$ hombres, $64,8 \%$ morenos, $54,5 \%$ con baja escolaridad, $53,4 \%$ residentes en Teresina y $70,9 \%$ por transmisión heterosexual. Conclusión e implicaciones para la práctica: hubo tendencia de crecimiento en los casos de VIH/SIDA en ancianos, en Piauí, durante los últimos 10 años. Los resultados de este estudio contribuyen al conocimiento de la dinámica epidemiológica de esta condición en el estado y al desarrollo de estrategias para prevención y control de la infección.

Palabras clave: Anciano; Notificación;VIH; Síndrome de Inmunodeficiencia Adquirida; Enfermedades de Transmisión Sexual; Epidemiología 


\section{INTRODUÇÃO}

O envelhecimento da população é uma realidade vivenciada por vários países do mundo. No Brasil, projetou-se aumento notório, de forma rápida e proporcional e, nos dias atuais, o grupo de idosos representa percentual importante da população brasileira, sendo que nos próximos 20 anos, essa proporção representará $13 \%$ da população, ultrapassando os 30 milhões. ${ }^{1}$

O processo de envelhecimento populacional foi resultado do aumento da expectativa de vida e da diminuição da taxa de natalidade, que associados à melhoria da qualidade de vida, resultante da evolução de tecnologias na área da saúde, permitiram aos idosos um envelhecimento saudável e ativo, com independência e autonomia para realização das necessidades básicas. ${ }^{2}$

Entretanto, alguns acometimentos limitantes à saúde dessa população ganharam destaque nos últimos anos. Em 2017, no Brasil, foram diagnosticados 42.420 novos casos de infecção pelo Vírus da Imunodeficiência Humana (HIV) e 37.971 casos de Síndrome da Imunodeficiência Adquirida (AIDS), sendo que a taxa por detecção por 100 mil habitantes foi de 18,3. Assim, no período de 1980 a junho de 2018, totalizaram-se 982.129 casos de Aids no País. No período entre 2007 e junho de 2018, as notificações no Sistema de Informação de Agravos de Notificação (SINAN) contabilizaram 247.795 casos de infecção pelo HIV no Brasil. Dentre esse percentual total, pessoas com idade igual ou superior a 60 anos apresentaram estimativa de 7.469 casos $(3,0 \%){ }^{3}$

Nas últimas três décadas, estimou-se para faixa etária senil 13.665 casos de Aids, com 34,36\% das ocorrências acometendo o sexo feminino. Percebe-se, portanto, que o delineamento epidemiológico denota necessidade de mudança da concepção social abordada ao idoso, cabendo a este estereótipo de inatividade sexual, o que reconfigura a essa população o risco para adquirir HIV, fato que contribui para o aumento expressivo do número de eventos. ${ }^{4}$

Informe Epidemiológico contabilizou casos encerrados até janeiro de 2020, no período de 2009 a 2019, 7.204 novos diagnósticos de Aids no Piauí, sendo 873 de 50 a 64 anos; 125 , de 65 a 79 anos; e 11 casos em pessoas com 80 anos ou mais. ${ }^{5}$

A ampliação do número de casos de infecção pelo HIV na população idosa se relaciona a alguns fatores: prolongamento da sobrevida, devido à terapia antirretroviral; escassez de campanhas direcionadas à prática sexual segura entre idosos, como a utilização de preservativos; além da existência de tabu sobre a sexualidade na velhice. Ademais, destaca-se a carência de conhecimento nesse segmento a respeito da patologia pelo próprio idoso. ${ }^{4,6}$

Diante do exposto, faz-se necessário que gestores e profissionais de saúde compreendam a importância de analisar o desenho epidemiológico dos casos de idosos com HIV/Aids, no estado do Piauí, Brasil, por meio dos registros de notificação no SINAN, para elaborarem estratégias de enfrentamento frente ao processo descrito. O conhecimento do perfil sociodemográfico, clínico e epidemiológico dos casos de HIV/Aids nesse grupo populacional fornecerá subsídios para elaboração e implementação de ações e políticas com enfoque preventivo e manejo do HIV em idosos.
Com base nessas considerações, objetivou-se analisar o perfil sociodemográfico, clínico e epidemiológico dos casos de HIV/ Aids em idosos no estado do Piauí.

\section{MÉTODO}

Trata-se de estudo descritivo, com coleta retrospectiva, em que se analisaram os casos notificados de HIV/Aids em idosos, no estado do Piauí, Brasil, no período de 2008 a 2018, representando série temporal dos casos entre os idosos, associando com o processo consistente do envelhecimento da população brasileira.

No ano de 2018, o Piauí contava com população estimada de 3.263.754 pessoas, desta, a de idosos estabelecida foi de 401.089 , representando $12,29 \%$ da população geral. ${ }^{7}$

Os dados foram obtidos do SINAN da Secretaria de Estado da Saúde do Piauí (SESAPI), na Coordenação de Doenças Transmissíveis, de agosto a setembro de 2019.

A população fonte do estudo foi constituída por todos os casos de HIV/Aids em adultos do estado do Piauí, Brasil, notificados no SINAN, no período de 2008 a 2018, correspondendo a 5.587 registros. Definiram-se como critérios de inclusão: idade igual ou maior que 60 anos, ser residente no Piauí e apresentar dados completos. Excluíram-se os casos em duplicidade. Alcançou-se, portanto, a amostra de 262 casos.

Para coleta de dados, utilizou-se de formulário elaborado especificamente para pesquisa e com base nas informações da ficha de notificação/investigação Aids (pacientes com 13 anos ou mais), registrada no SINAN, que possuía as variáveis abordadas neste estudo: idade, sexo, raça, escolaridade, município de residência, critérios de definição da doença, segundo Critério Rio de Janeiro/Caracas e CDC Adaptado, modo de transmissão e evolução do tratamento.

A variável idade foi categorizada, agrupando-se em intervalos de 10 anos, nas faixas etárias 60-69 anos, 70-79 anos e 80 anos e mais, para classificar os idosos em jovens, meio idosos e idosos muito idosos, ${ }^{8}$ respectivamente, mesmas faixas etárias disponíveis no SINAN.

Para processamento dos dados, utilizou-se do programa gratuito TabWin (Tab para Windows), versão 3.6, software desenvolvido pelo Departamento de Informática do SUS (DATASUS).

Para análise dos dados, elaboraram-se relatórios com distribuições de frequência dos casos de HIV/Aids e, para calcular as frequências absoluta e relativa e a incidência, utilizouse do programa Excel, versão 7.0. Calculou-se o coeficiente de incidência de cada ano para verificar como o HIV/Aids se comportou na população acima de 60 anos no Piauí.

Em relação aos aspectos éticos, solicitou-se autorização da SESAPI, e após autorização institucional, foi aprovado pelo Comitê de Ética em Pesquisa da Universidade Federal do Piauí, conforme parecer no 3.335.061 e Certificado de Apresentação para Apreciação Ética (CAAE) 14072819.3.0000.5214, sendo assegurados o anonimato e sigilo das informações pessoais acessadas e o compromisso do uso dos dados para fins da pesquisa. 


\section{RESULTADOS}

No estudo, analisaram-se os 262 casos novos de HIV/ Aids em idosos notificados no SINAN, no período de 2008 a 2018, no Piauí, Brasil. Na Tabela 1, estão apresentados os dados em relação ao perfil sociodemográfico dos casos. Em relação à faixa etária, o grupo mais acometido foi aquele com idade entre 60 e 69 anos (76,0\%), maioria do sexo masculino $(64,9 \%)$, pardos $(64,8 \%)$, com baixa escolaridade $(54,5 \%)$, seguido dos idosos sem escolaridade (14,1\%). Quanto ao município de residência, 117 (53,4\%) eram de Teresina, capital piauiense. Destaca-se o alto percentual de ignorados na variável escolaridade $(14,9 \%)$.

Dos 5.849 casos novos de HIV/Aids em adultos notificados no SINAN, no período de 2008 a 2018, 262 ocorreram com idosos, representando 4,5\% do número total de casos. Embora esse percentual não seja tão expressivo, verifica-se aumento no número de pessoas notificadas com HIV/Aids de um ano para outro, com exceção dos anos de 2011, 2014 e 2018 que registraram aumento de casos em comparação aos anos anteriores (Tabela 2).

A Figura 1 apresenta o coeficiente de incidência de HIV/Aids em idosos, em cada ano, em que se verificou maior coeficiente no ano de 2017, de 9,6 casos por $100 \mathrm{mil}$ habitantes, seguido do ano de 2015 , com 8,2 casos por 100 mil habitantes.

$\mathrm{Na}$ Tabela 3, encontram-se os dados referentes às características clínicas e epidemiológicas dos casos analisados. Dos 262 casos analisados, apenas 190 preencheram os critérios de definição, sendo também observado percentual significativo de ignorados $(21,9 \%)$, no modo de transmissão. Destaca-se que em $119(62,7 \%)$ dos casos foi utilizado o critério do CDC Adaptado, em que $186(70,9 \%)$ tiveram como modo de transmissão a relação heterossexual e 204 (77,9\%) evoluíram vivos e 47 (17,9\%) com óbito por Aids (Tabela 3).

Na Figura 2, tem-se aumento do número de notificações de casos novos, nas três faixas etárias, ao longo do período analisado.

Tabela 1. Perfil sociodemográfico dos casos notificados de HIV/Aids em idosos, no período de 2008 a 2018. Teresina, PI, Brasil, 2019. $(n=262)$

\begin{tabular}{|c|c|c|c|c|c|c|c|c|c|c|c|c|c|}
\hline Variáveis & 2008 & 2009 & 2010 & 2011 & 2012 & 2013 & 2014 & 2015 & 2016 & 2017 & 2018 & $\begin{array}{l}\text { Total } \\
\text { Abs.* }\end{array}$ & $\begin{array}{c}\text { Total } \\
(\%)\end{array}$ \\
\hline \multicolumn{14}{|l|}{ Idade } \\
\hline $60-69$ & 8 & 8 & 13 & 12 & 16 & 24 & 14 & 23 & 27 & 33 & 21 & 199 & 76,0 \\
\hline 70- 79 & 3 & 2 & 7 & 2 & 6 & 1 & 5 & 8 & 3 & 10 & 5 & 52 & 19,8 \\
\hline$\geq 80$ & - & 1 & - & 1 & - & - & 3 & 1 & 2 & 1 & 2 & 11 & 4,2 \\
\hline Total Abs.* & 11 & 11 & 20 & 15 & 22 & 25 & 22 & 32 & 32 & 44 & 28 & 262 & 100,0 \\
\hline Total (\%) & 4,2 & 4,2 & 7,6 & 5,7 & 8,4 & 9,5 & 8,4 & 12,2 & 12,2 & 16,8 & 10,8 & 100,0 & - \\
\hline \multicolumn{14}{|l|}{ Sexo } \\
\hline Masculino & 7 & 7 & 16 & 10 & 11 & 14 & 14 & 24 & 19 & 30 & 18 & 170 & 64,9 \\
\hline Feminino & 4 & 4 & 4 & 5 & 11 & 11 & 8 & 8 & 13 & 14 & 10 & 92 & 35,1 \\
\hline Total Abs.* & 11 & 11 & 20 & 15 & 22 & 25 & 22 & 32 & 32 & 44 & 28 & 262 & 100,0 \\
\hline Total (\%) & 4,2 & 4,2 & 7,6 & 5,7 & 8,4 & 9,5 & 8,4 & 12,2 & 12,2 & 16,8 & 10,8 & 100,0 & - \\
\hline \multicolumn{14}{|l|}{ Raça } \\
\hline Branca & 1 & 4 & 6 & 1 & 4 & 5 & 1 & 5 & 6 & 12 & 3 & 48 & 18,3 \\
\hline Preta & 4 & 1 & 1 & 1 & 3 & 6 & 3 & 2 & 5 & 5 & 5 & 36 & 13,7 \\
\hline Amarela & - & - & - & - & - & - & - & - & - & - & - & - & 0,0 \\
\hline Parda & 6 & 5 & 13 & 12 & 15 & 13 & 18 & 25 & 19 & 24 & 20 & 170 & 64,9 \\
\hline Indígena & - & - & - & 1 & - & - & - & - & - & - & - & 1 & 0,4 \\
\hline Ignorado & - & 1 & - & - & - & 1 & - & - & 2 & 3 & - & 7 & 2,7 \\
\hline Total Abs.* & 11 & 11 & 20 & 15 & 22 & 25 & 22 & 32 & 32 & 44 & 28 & 262 & 100,0 \\
\hline Total (\%) & 4,2 & 4,2 & 7,6 & 5,7 & 8,4 & 9,5 & 8,4 & 12,2 & 12,2 & 16,8 & 10,8 & 100,0 & - \\
\hline
\end{tabular}

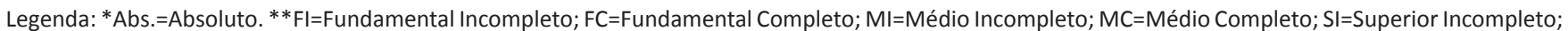
SC=Superior Completo. ${ }^{* * *}=219$ casos por município de residência, não de notificação.

Fonte: SINAN/SESAPI (2019) 
Tabela 1. Continuação...

\begin{tabular}{|c|c|c|c|c|c|c|c|c|c|c|c|c|c|}
\hline Variáveis & 2008 & 2009 & 2010 & 2011 & 2012 & 2013 & 2014 & 2015 & 2016 & 2017 & 2018 & $\begin{array}{l}\text { Total } \\
\text { Abs.* }\end{array}$ & $\begin{array}{c}\text { Total } \\
(\%)\end{array}$ \\
\hline \multicolumn{14}{|l|}{ Escolaridade** } \\
\hline Sem & 3 & 2 & 7 & 2 & 1 & 5 & 3 & 2 & 2 & 6 & 4 & 37 & 14,1 \\
\hline $\mathrm{FI}$ & 5 & 4 & 6 & 6 & 7 & 7 & 6 & 7 & 10 & 13 & 4 & 75 & 28,6 \\
\hline FC & - & 1 & 7 & 3 & 5 & 7 & 6 & 12 & 5 & 13 & 9 & 68 & 26,0 \\
\hline $\mathrm{Ml}$ & - & - & - & 2 & 3 & 3 & - & 1 & 4 & 1 & 4 & 18 & 6,9 \\
\hline $\mathrm{MC}$ & 1 & 1 & - & 1 & 4 & 1 & 3 & - & 2 & 1 & 2 & 16 & 6,1 \\
\hline SI & - & - & - & - & - & - & - & 1 & - & 1 & - & 2 & 0,8 \\
\hline SC & - & - & - & - & - & 1 & - & 1 & 1 & 3 & 1 & 7 & 2,7 \\
\hline Ignorado & 2 & 3 & - & 1 & 2 & 1 & 4 & 8 & 8 & 6 & 4 & 39 & 14,8 \\
\hline Total Abs.* & 11 & 11 & 20 & 15 & 22 & 25 & 22 & 32 & 32 & 44 & 28 & 262 & 100,0 \\
\hline Total (\%) & 4,2 & 4,2 & 7,6 & 5,7 & 8,4 & 9,5 & 8,4 & 12,2 & 12,2 & 16,8 & 10,8 & 100,0 & - \\
\hline \multicolumn{14}{|l|}{$\begin{array}{l}\text { Município } \\
\text { residência*** }\end{array}$} \\
\hline Teresina & 3 & 3 & 6 & 3 & 10 & 13 & 11 & 12 & 18 & 25 & 13 & 117 & 53,4 \\
\hline Mac. Litoral & 1 & 1 & 1 & 3 & 3 & 2 & 2 & 5 & 3 & 2 & 2 & 25 & 11,4 \\
\hline $\begin{array}{l}\text { Mac. Meio } \\
\text { Norte }\end{array}$ & 3 & - & 3 & 3 & 5 & 3 & 2 & 2 & 5 & 1 & 2 & 29 & 13,2 \\
\hline $\begin{array}{l}\text { Mac. Semi } \\
\text { Árido }\end{array}$ & 1 & - & 3 & 2 & 2 & 2 & 2 & 7 & 2 & 7 & 5 & 33 & 15,1 \\
\hline Mac. Cerrados & - & & 4 & 1 & 4 & 1 & - & - & 2 & 2 & 1 & 15 & 6,9 \\
\hline Total Abs. * & 8 & 4 & 17 & 12 & 24 & 21 & 17 & 26 & 30 & 37 & 23 & 219 & 100,0 \\
\hline Total (\%) & 3,1 & 1,5 & 6,5 & 4,6 & 9,2 & 8,0 & 6,5 & 9,9 & 11,5 & 14,1 & 8,8 & 100,0 & - \\
\hline
\end{tabular}

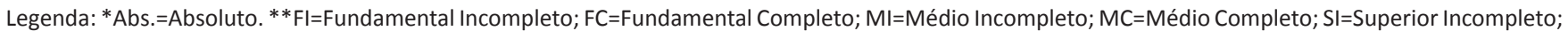
SC=Superior Completo. ${ }^{* *}=219$ casos por município de residência, não de notificação.

Fonte: SINAN/SESAPI (2019)

Tabela 2. Distribuição dos casos de HIV/Aids, segundo ano de diagnóstico e faixa etária, no período de 2008 a 2018 . Teresina, PI, Brasil, 2019. ( $n=262)$

\begin{tabular}{|c|c|c|c|}
\hline \multirow{2}{*}{ Ano de Diagnóstico } & $<60$ anos & $>60$ anos & Total \\
\hline & $\mathrm{n}(\%)$ & $\mathrm{n}(\%)$ & $\mathbf{N}$ \\
\hline 2008 & $264(96,0)$ & $11(4,0)$ & 275 \\
\hline 2009 & $311(96,6)$ & $11(3,4)$ & 322 \\
\hline 2010 & $362(94,8)$ & $20(5,2)$ & 382 \\
\hline 2011 & $302(95,3)$ & $15(4,7)$ & 317 \\
\hline 2012 & $436(95,2)$ & $22(4,8)$ & 458 \\
\hline 2013 & $493(95,2)$ & $25(4,8)$ & 518 \\
\hline 2014 & $590(96,4)$ & $22(3,6)$ & 612 \\
\hline 2015 & $569(94,7)$ & $32(5,3)$ & 601 \\
\hline 2016 & $594(94,9)$ & $32(5,1)$ & 626 \\
\hline 2017 & $801(94,8)$ & $44(5,2)$ & 845 \\
\hline 2018 & $865(96,9)$ & $28(3,1)$ & 893 \\
\hline Total & $5.587(95,5)$ & $262(4,5)$ & 5.849 \\
\hline
\end{tabular}

Fonte: SINAN/SESAPI (2019). 
Tabela 3. Perfil clínico e epidemiológico dos casos notificados de HIV/Aids em idosos, no período de 2008 a 2018. Teresina, PI, Brasil, 2019. $(n=262)$

Variáveis

2008-2018*

Total Total

Abs.** (\%)

\begin{tabular}{|c|c|c|c|c|c|c|c|c|c|c|c|c|c|}
\hline \multicolumn{14}{|l|}{$\begin{array}{l}\text { Critérios } \\
\text { definição*** }\end{array}$} \\
\hline CDC Adaptado & 5 & 9 & 11 & 7 & 16 & 16 & 11 & 13 & 11 & 14 & 6 & 119 & 62,6 \\
\hline RJ/Caracas & 5 & - & 8 & 6 & 6 & 8 & 7 & 9 & 12 & 6 & 4 & 71 & 37,4 \\
\hline Total Abs.** & 10 & 9 & 19 & 13 & 22 & 24 & 18 & 22 & 23 & 20 & 10 & 190 & 100,0 \\
\hline Total (\%) & 5,3 & 4,7 & 10,0 & 6,8 & 11,6 & 12,6 & 9,5 & 11,6 & 12,1 & 10,5 & 5,3 & 100,0 & - \\
\hline \multicolumn{14}{|l|}{ Modo transmissão } \\
\hline Homossexual & 1 & - & 1 & - & - & 2 & 1 & 2 & 1 & - & 1 & 9 & 3,4 \\
\hline Bissexual & - & - & 1 & - & 1 & - & - & 1 & - & 3 & - & 6 & 2,3 \\
\hline Heterossexual & 8 & 10 & 15 & 11 & 17 & 17 & 16 & 21 & 21 & 25 & 25 & 186 & 70,9 \\
\hline Drogas & - & - & - & - & - & - & - & - & - & 1 & - & 1 & 0,4 \\
\hline Perinatal & - & - & - & - & - & 1 & 1 & - & - & 1 & - & 3 & 1,1 \\
\hline Ignorado & 2 & 1 & 3 & 4 & 4 & 5 & 4 & 8 & 10 & 14 & 2 & 57 & 21,9 \\
\hline Total Abs.** & 11 & 11 & 20 & 15 & 22 & 25 & 22 & 32 & 32 & 44 & 28 & 262 & 100,0 \\
\hline Total (\%) & 4,2 & 4,2 & 7,6 & 5,7 & 8,4 & 9,6 & 8,4 & 12,2 & 12,2 & 16,8 & 10,7 & 100,0 & - \\
\hline \multicolumn{14}{|l|}{ Evolução } \\
\hline Vivo & 8 & 11 & 15 & 11 & 17 & 20 & 18 & 24 & 24 & 31 & 25 & 204 & 77,9 \\
\hline Óbito por Aids & 3 & - & 4 & 3 & 4 & 5 & 4 & 6 & 8 & 7 & 3 & 47 & 17,9 \\
\hline Óbito outras causas & - & - & - & - & 1 & - & - & 2 & - & - & - & 3 & 1,1 \\
\hline Ignorado & - & - & 1 & 1 & - & - & - & - & - & 6 & - & 8 & 3,1 \\
\hline Total Abs. ${ }^{* *}$ & 11 & 11 & 20 & 15 & 22 & 25 & 22 & 32 & 32 & 44 & 28 & 262 & 100,0 \\
\hline Total (\%) & 4,2 & 4,2 & 7,6 & 5,7 & 8,4 & 9,6 & 8,4 & 12,2 & 12,2 & 16,8 & 10,7 & 100,0 & - \\
\hline
\end{tabular}

Legenda: *Cada coluna corresponde a um ano, seguindo sequência: 2008, 2009, 2010, 2011, 2012, 2013, 2014, 2015, 2016, 2017 e 2018. **Abs.=Absoluto. $* * *=\mathrm{n}$ 190. Fonte: SINAN/SESAPI (2019).

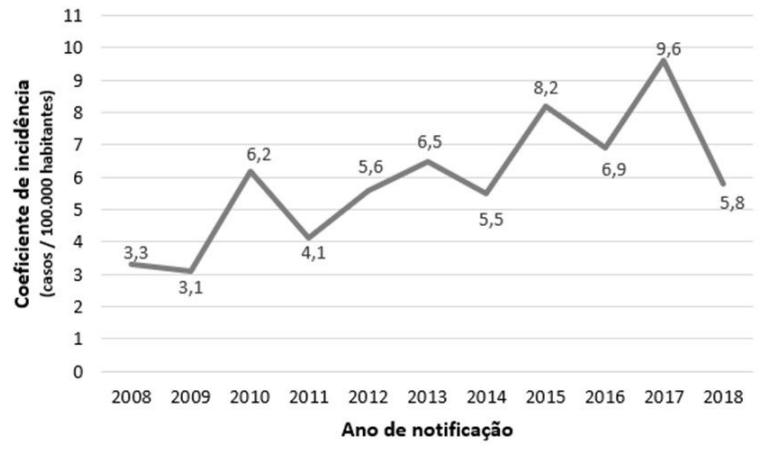

Figura 1. Coeficiente de incidência (por 100 mil habitantes) de HIV/Aids em idosos de 2008 a 2018, segundo ano de notificação. Teresina, PI, Brasil, 2019.

Fonte: SINAN/SESAPI (2019)

\section{DISCUSSÃO}

A tendência de aumento do número de casos de HIV/Aids em idosos, no Piauí, registrada neste estudo, corrobora com estudos nacionais, em que se constata esse crescimento

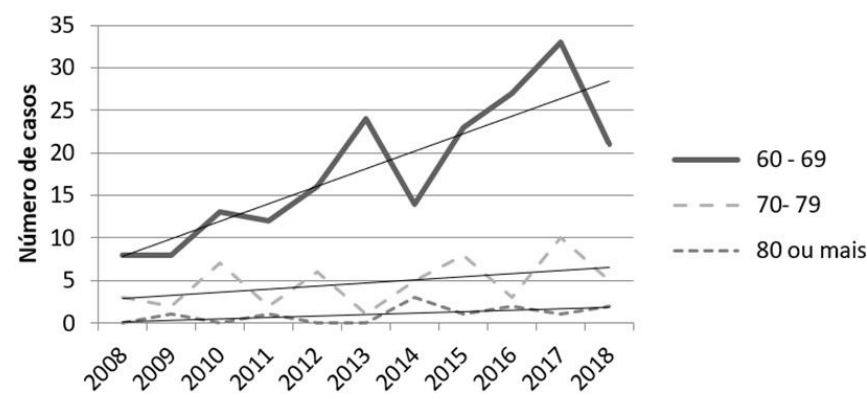

Figura 2. Casos novos e tendência de HIV/Aids em idosos de 2008 a 2019, segundo faixa etária e ano de notificação. Teresina, PI, Brasil, 2019.

Fonte: SINAN/SESAPI (2019)

relacionado ao aumento da expectativa de vida e ao não uso de medidas preventivas para prática de sexo seguro pelos idosos, que passaram a ter vida sexual mais ativa, devido ao uso de medicamentos para impotência sexual e reposição hormonal. ${ }^{9,10}$ 
Verificou-se que no Piauí, ao longo do período analisado, em alguns anos, houve queda do número de casos notificados na população acima de 60 anos, diferentemente do que ocorreu no Brasil, em que se observa, no mesmo período, aumento crescente e contínuo de casos de HIV/Aids notificados no SINAN. ${ }^{3}$ Destaca-se que os casos notificados no SINAN são eventos novos de HIV/Aids, conforme período determinado, não sendo possível realizar análise epidemiológica mais rigorosa com relação às tendências da infecção pelo HIV no estado pesquisado, considerando que a notificação compulsória da infecção pelo HIV data de 2014 e ainda há subnotificação.

No Brasil, segundo o último Boletim Epidemiológico de HIV/ Aids, a taxa de detecção da Aids vem decrescendo nos últimos anos, mas as Regiões Norte e Nordeste apresentam tendência de detecção maiores, sendo que, no Piauí, no período de 2007 a 2017 , houve aumento dessa taxa de $23 \%$, superior a nacional que foi de 18,3/100 mil habitantes. ${ }^{3}$ Esse comportamento foi verificado no presente estudo que mostrou tendência de crescimento do número de casos novos notificados. O fenômeno, portanto, quando analisado a nível nacional, reforça os registros encontrados no estado, observando-se crescimento tendencioso e indicativo de aumento tímido do acesso da população aos sistemas de saúde. ${ }^{11}$

Em relação ao perfil sociodemográfico analisado, a faixa etária mais acometida foi entre 60 e 69 anos, considerados idosos jovens, o que pressupõe vida sexual ativa, haja vista que quanto mais avançada a idade, menor importância é atribuída pelos idosos à prática sexual, devido às alterações fisiológicas e biológicas em relação à disposição do idoso em manter relação sexual, presença de patologias e preconceito por parte da família. ${ }^{12}$ Além disso, com os avanços da ciência, essa parcela da população tem mantido as práticas sexuais com o passar dos anos. A reposição hormonal e as medicações para melhorar o desempenho sexual possibilitaram uma vida sexual mais ativa, mas também tornaram os idosos mais susceptíveis a contraírem o HIV, em virtude de práticas sexuais desprotegidas, associadas a conceitos errados sobre o uso de preservativo, sendo realidade estendida a todas as idades, não apenas aos idosos. ${ }^{13}$

Infere-se, ainda, que a infecção ocorreu anos antes, na faixa etária de 50 a 60 anos, uma vez que para pessoa ser considerada um caso de Aids, é necessário intervalo de cinco a 10 anos do momento da transmissão à situação de soropositivo. ${ }^{14}$ Por isso, a importância da educação em saúde em todas as faixas etárias, como instrumento fundamental, no processo de prevenção, controle e tratamento da infecção pelo vírus do HIV.

Quanto ao sexo, o mais afetado foi o masculino. Esses números são ratificados pelo Boletim Epidemiológico do Ministério da Saúde, que mostra que o número de casos de HIV/Aids, no Brasil, é maior entre os homens. ${ }^{3}$ Como razões, pode-se apontar o preconceito enraizado dos homens quanto à ida ao serviço de saúde e ao uso inadequado do preservativo, justificado pela alegação de perda de prazer sexual com o uso. ${ }^{15}$ Esse estigma se apresenta em potencial, com efeitos sobre a identidade dos indivíduos, dos grupos e das relações sociais, além do reflexo nos processos de saúde e adoecimento. ${ }^{16}$

Entre as mulheres, verifica-se, de modo geral que, nos anos de 2007 a 2017, a taxa de detecção apresentou queda em quase todas as faixas etárias, exceto na de 60 anos ou mais, na qual foi observado aumento de $21,2 \% .{ }^{3}$ Apesar do sexo masculino ter sido o mais acometido, no presente estudo, o sexo feminino também apresentou resultado significativo, destacando o fenômeno da feminização do HIV/Aids. Esse processo pode ser explicado pela maior vulnerabilidade biológica e social que a mulher apresenta, aquele se refere às alterações morfofisiológicas; a social é relativa à persistência de padrões cultural e religioso que afetam negativamente a adoção de medidas preventivas, principalmente, quando se trata da população idosa. ${ }^{17}$

No tocante à raça, a parda apresentou maior significância entre os resultados. Alguns estudos apontam maior prevalência na raça branca, enquanto outros confrontam com evidência em indivíduos pardos. No Brasil, houve crescimento de 33,5\% entre as pessoas autodeclaradas pardas, no período de 2007 a 2017. ${ }^{3}$ Mas, é necessário enfatizar que a variável possui aspecto subjetivo, uma vez que é respondida pelo próprio indivíduo, podendo, também, ser baseada na interpretação do profissional que está preenchendo a ficha. ${ }^{4}$

Com relação à escolaridade, no mesmo período, verificou-se elevado percentual de casos ignorados, o que dificulta melhor avaliação dos casos de HIV/Aids relativos a esse item. Quanto aos casos com escolaridade informada, o presente estudo constatou que a baixa escolaridade, seguida dos sem escolaridade, apresentou as maiores porcentagens, o que pode interferir na compreensão dos riscos da doença e dos métodos preventivos por parte dos idosos participantes. Por isso, o grau de escolaridade tem grande impacto sobre a transmissão das infecções sexualmente transmissíveis. ${ }^{18}$ Há, também, associação à dificuldade na adesão ao tratamento e ao baixo conhecimento acerca da cadeia de transmissão do HIV. ${ }^{19}$ Dessa forma, a alta escolaridade é uma variável importante na prevenção das infecções, dentre elas o HIV/Aids, considerando que pessoas com menor tempo de estudo tendem a valorizar menos os cuidados à saúde.

Quanto ao município de residência, Teresina, capital do Piauí, obteve o maior percentual de casos notificados, o que pode ser explicado pelo fato de ser o município mais populoso e considerado grande polo de saúde, o que, consequentemente, traz facilidade de acesso ao sistema de saúde para diagnóstico e notificação. Ainda, são poucos os estudos que abordam essa temática no Piauí, desta forma, não há comparativos. Observa-se que as Regiões Norte, Nordeste e Centro-Oeste tiveram notável aumento das incidências, ${ }^{20}$ devido à mudança epidemiológica, com a expansão da epidemia para outras áreas de abrangência. Pois, durante as décadas de 1980 e 1990, a epidemia se manteve basicamente restrita às Regiões Sudeste e Sul. ${ }^{21}$

Para fins de vigilância epidemiológica, os critérios de definição CDC Adaptado e Rio de Janeiro/Caracas definem caso de Aids em indivíduos com idades acima de 13 anos, sendo que, neste 
estudo, o CDC Adaptado apresentou percentual maior, o que pode ser explicado pelo fato de ser fundamentado na evidência laboratorial de infecção pelo HIV e na presença de doenças indicativas de Aids. Ao contrário, o critério Rio de Janeiro/ Caracas é baseado na identificação clínica de sinais e sintomas de doenças variadas. ${ }^{22}$ Não se encontram na literatura estudos comparativos com essa determinada variável.

O modo de transmissão mais prevalente foi o heterossexual, ou seja, pela via sexual, situação também confirmada em outras pesquisas ${ }^{4,23}$ que apontaram a prática sexual desprotegida como principal via de transmissão entre as pessoas com mais de 60 anos, apesar da transmissão homo/bissexual entre homens ser também relevante, o que está associado à baixa adesão ao uso de preservativo por razões culturais e à submissão da população feminina ao cônjuge. Entende-se, portanto, que os idosos apresentam vida sexual ativa e isso deve ser considerado pelos profissionais de saúde na abordagem dessa população $e$ nas campanhas de prevenção de HIV/Aids. ${ }^{1}$

Então, a vulnerabilidade dos idosos às infecções sexualmente transmissíveis pode ter influência de vários fatores, desde o comportamento de risco até as lacunas no manejo correto dos profissionais da saúde à questão. Dessa forma, o investimento na capacitação dos profissionais para abordagem adequada da percepção de risco pelos idosos e a necessidade de adotar comportamentos seguros podem trazer consequências positivas para diminuição dos índices. ${ }^{24}$

A maioria dos casos evoluiu viva. Estudo enfatiza a abordagem clínico-terapêutica do HIV como fator determinante na mudança do panorama da infecção. O tratamento é objetivo no prolongamento da sobrevida e melhora da qualidade de vida, por meio da redução da carga viral e, consequentemente, reconstituição do sistema imunológico. ${ }^{25}$

A limitação do estudo deteve-se ao alto percentual de informações ignoradas, o que pode estar relacionado ao preenchimento inadequado ou negligente das fichas. Além disso, a realização da pesquisa, a partir de fontes secundárias, pode ter algum prejuízo na qualidade dos dados obtidos, em face de possíveis inconsistências. Todavia, tomou-se o cuidado de excluir do estudo os casos duplicados ou com dados incompletos. Acrescenta-se que o SINAN, de onde foram extraídos os dados desta pesquisa, é a base de informações oficiais de morbidade no Brasil. Portanto, importante ferramenta para pesquisas epidemiológicas.

\section{CONCLUSÃO}

Os resultados mostram que os idosos fazem parte de grupo de risco em ascensão, no estado do Piauí, considerando que o número de casos de HIV/Aids nessa população apresentou tendência de crescimento. Nos casos notificados de idosos com HIV/Aids, no período de 2008 a 2018, a maioria era do sexo masculino, com idades entre 60 e 69 anos, apresentando baixo nível de escolaridade, tendo como forma de transmissão o modo heterossexual.
Os resultados contribuem para o conhecimento sobre a dinâmica epidemiológica desse agravo no estado pesquisado e a avaliação das estratégias de prevenção e controle da infecção. Poderá, ainda, subsidiar o desenvolvimento de ações e estratégias por gestores e equipe de saúde que visem reduzir a vulnerabilidade desse grupo populacional frente à doença, como elaboração de políticas públicas com especificidades voltados às pessoas idosas com HIV/Aids, melhoria do acesso aos serviços de saúde e, consequentemente, às estratégias de prevenção e controle, aos meios de diagnóstico e tratamento, além de aumento da adesão à terapia. No entanto, ainda é necessária a realização de novas pesquisas para levantamento dos fatores relacionados à vulnerabilidade dos idosos à infecção.

\section{AGRADECIMENTOS}

À Coordenação Estadual de Doenças Transmissíveis IST/ Aids, da Secretaria do Estado do Piauí (SESAPI).

\section{CONTRIBUIÇÕES DOS AUTORES}

Desenho do estudo. Chrystiany Plácido de Brito Vieira. Ana Clara dos Santos e Silva Costa. Maria do Carmo Lopes Dias. Telma Maria Evangelista de Araújo.

Coleta de dados. Chrystiany Plácido de Brito Vieira. Ana Clara dos Santos e Silva Costa. Maria do Carmo Lopes Dias.

Análise de dados e interpretação dos resultados. Chrystiany Plácido de Brito Vieira. Ana Clara dos Santos e Silva Costa. Maria do Carmo Lopes Dias. Telma Maria Evangelista de Araújo. Francisca Tereza de Galiza.

Redação e revisão crítica do manuscrito. Aprovação da versão final do artigo. Responsabilidade por todos os aspectos do conteúdo e a integridade do artigo publicado. Chrystiany Plácido de Brito Vieira. Ana Clara dos Santos e Silva Costa. Maria do Carmo Lopes Dias. Telma Maria Evangelista de Araújo. Francisca Tereza de Galiza.

\section{EDITOR ASSOCIADO}

Gerson Luiz Marinho

\section{REFERÊNCIAS}

1. Instituto Brasileiro de Geografia e Estatística. Síntese de indicadores sociais: uma análise das condições de vida da população brasileira - 2010 [Internet]. Rio de Janeiro: IBGE; 2010 [citado 2019 jan 10]. Disponível em: http://www.ibge.gov.br/home/estatistica/populacao/ condicaodevida/indicadoresmini mos/sinteseindicsociais2010/default. $\mathrm{htm}$

2. Bittencourt GKGD, Moreira MASP, Meira LCS, Nóbrega MM, Nogueira JA, Silva AO. Beliefs of older adults about their vulnerability to HIV/ Aids, for the construction of nursing diagnoses. Rev Bras Enferm. 2015;68(4):579-85. http://dx.doi.org/10.1590/0034-7167.2015680402i. PMid:26422027.

3. Ministério da Saúde (BR), Secretaria de Vigilância em Saúde. Boletim Epidemiológico HIV/Aids. Brasília: Ministério da Saúde; 2018.

4. Alencar RA, Silva AG, Cavalcanti V, Santos TS, Bragagnollo GR, Santos $\mathrm{KS}$ et al. Repply: integrative review of literature: nursing care to aged 
people with HIV. Rev Bras Enferm. 2018;71(Suppl 5):2079-80. http:// dx.doi.org/10.1590/0034-7167.2018710502c. PMid:30365767.

5. Piauí, Secretaria de Saúde, Coordenação de Doenças Transmissíveis. Informe epidemiológico da Aids no Piauí [Internet]. Teresina: SESAPI; 2020 [citado 2019 jan 10]. Disponível em: http://www.saude.pi.gov.br/ uploads/warning_document/file/456/ACFrOgAtGEdMzOsXzCCzJdSsOpqYhratjeswy1cZUOMvbaMXfGZB2rBPoVppBQyvj99r6S-_n8rWnEm7Q-vH4HBiopHbjcu7AtP8Bs4v2iKjW_jO7ICWn4IGJPtRC0A_.pdf

6. Maia DC, Zanin L, Silva ASF, Ambrosano GMB, Flório FM. Notification of cases of HIV/AIDS among the elderly in the state of Ceará: the historical sequence between 2005 and 2014. Rev Bras Geriatr Gerontol. 2018;21(5):542-52. http://dx.doi.org/10.1590/1981-22562018021.180041.

7. Instituto Brasileiro de Geografia e Estatística. Síntese de indicadores sociais: uma análise das condições de vida da população brasileira - 2018 [Internet]. Rio de Janeiro: IBGE; 2018 [citado 2019 jan 10]. Disponível em: http://www.ibge.gov.br/home/estatistica/populacao/condicaodevida/ indicadoresmini mos/sinteseindicsociais2018/default.htm

8. Veras RP. País jovem com cabelos brancos: a saúde do idoso no Brasil. Rio de Janeiro: Relume Dumará; 1994.

9. Silva MM, Vasconcelos ALR, Ribeiro LKNP. Caracterização epidemiológica dos casos de AIDS em pessoas com 60 anos ou mais, Pernambuco, Brasil, 1998 a 2008. Cad Saude Publica. 2013;29(10):2131-5. http:// dx.doi.org/10.1590/0102-311X00161112. PMid:24127106.

10. Silva HR, Marreiros MC, Figueiredo TS, Figueiredo MLF. Características clínico-epidemiológicas de pacientes idosos com aids em hospital de referência, Teresina-PI, 1996 a 2009. Epidemiol Serv Saude. 2011;20(4):499507. http://dx.doi.org/10.5123/S1679-49742011000400009.

11. Piauiense JNF. Epidemiologia da HIV/AIDS em Teresina-PI: análise retrospectiva. Braz J Surg Clin Res [Internet]. 2018; [citado 2019 jan 10];21(3):7-12. Disponível em: https://www.mastereditora.com.br/ periodico/20180204_154640.pdf

12. Gois AB, Santos RFLS, Silva TPS, Aguiar VFF. Percepção do homem idoso em relação a sua sexualidade. Enferm Foco. 2017;8(3):14-8. http://dx.doi.org/10.21675/2357-707X.2017.v8.n3.1024.

13. Alencar RA, Ciosak SI. Late diagnosis and vulnerabilities of the elderly living with HIV/AIDS. Rev Esc Enferm USP. 2015;49(2):229-35. http:// dx.doi.org/10.1590/S0080-623420150000200007. PMid:25992821.

14. Oliveira MLC, Paz LC, Melo GF. Dez anos de epidemia do HIV-AIDS em maiores de 60 anos no Distrito Federal - Brasil. Rev Bras Epidemiol. 2013;16(1):30-9. http://dx.doi.org/10.1590/S1415-790X2013000100003. PMid:23681320.

15. Almeida DVJ, Pinheiro LMG. Epidemiologia dos Idosos com AIDS na Bahia segundo o SINAN de 2014 a 2016. Rev Mult Psic [Internet]. 2014; [citado 2019 jan 10];11(37):640-52. Disponível em: https://idonline. emnuvens.com.br/id/article/view/676

16. Cassétte JB, Silva LC, Felício EEAA, Soares LA, Morais RA, Prado TS et al. HIV/AIDS among the elderly: stigmas in healthcare work and training. Rev Bras Geriatr Gerontol. 2016;19(5):733-44. http://dx.doi. org/10.1590/1809-98232016019.150123.

17. Araújo APS, Bertolini SMMG, Bertolini DA. Perfil epidemiológico e imunológico de idosos infectados pelo vírus da imunodeficiência humana. Estud Interdiscipl Envelhec. 2015;20(1):121-38.

18. Viana PAS, Novais CT, Reis RWC, Flor SMC, Rosa PB. Aspectos epidemiológicos, clínicos e evolutivos da aids em idosos no norte do Ceará. Sanare [Internet].2017; [citado 2019 jan 10];16(2):31-6. Disponíve em: https://sanare.emnuvens.com.br/sanare/article/view/1175

19. Affeldt AB, Silveira MF, Barcelos RS. Perfil de pessoas idosas vivendo com HIV/aids em Pelotas, sul do Brasil, 1998 a 2013. Epidemio Serv Saude. 2015;24(1):79-86. http://dx.doi.org/10.5123/S167949742015000100009.

20. Silva KPBD, Tomasi LG, Elias RM, Silva LM. Perfil epidemiológico da infecção pelo vírus HIV na cidade de Várzea Grande-MT, no período de 2011 a 2014. Rev Eletr UNIVAG. 2016;15(15):57-69. http://dx.doi. org/10.18312/1980-7341.n15.2016.346.

21. Dantas CC, Dantas FC, Monteiro BAC, Leite JL. Perfil epidemiológico dos pacientes com HIV atendidos em um centro de saúde da região litorânea do estado de Rio de Janeiro, Brasil, 2010-2011. Arq Catarin Med [Internet]. 2017; [citado 2019 jan 10];46(1):22-32. Disponíve em: http://www.acm.org.br/acm/seer/index.php/arquivos/article/ view/250

22. Ministério da Saúde (BR), Secretaria de Vigilância em Saúde. Protocolo clínico e diretrizes terapêuticas para manejo da infecção pelo HIV em adulto. Brasília: Ministério da Saúde; 2018.

23. Jesus SMC, Caldas AJM, Correa RGCF, Soares DL, Pereira LFB, Aquino DMC. Características dos idosos com HIV/AIDS notificados no Estado do Maranhão. Rev Pesq Saúde [Internet]. 2014; [citado 2019 jan 10];15(2):276-9. Disponível em: http://www.periodicoseletronicos. ufma.br/index.php/revistahuufma/article/view/3264

24. Andrade J, Ayres JA, Alencar RA, Duarte MTC, Parada CMGL. Vulnerability of the elderly to sexually transmitted infections. Acta Paul Enferm. 2017;30(1):8-15. http://dx.doi.org/10.1590/1982-0194201700003.

25. Melo MC, Pimenta AM, Donalísio MR. Perfil epidemiológico de idosos com AIDS na macrorregião de saúde de Belo Horizonte. R Enferm Cent O Min [Internet]. 2016; [citado 2019 jan 10];1(6):202033. Disponível em: http://www.seer.ufsj.edu.br/index.php/recom/ article/view/330 